\title{
Microfluidic experimental setup for adhesion and recovery measurements of red blood cells in sickle cell disease
}

\author{
Kostyantyn R. Partola ${ }^{\mathrm{a}}$, Biree Andemariam ${ }^{\mathrm{b}}$, George Lykotrafitis ${ }^{\mathrm{a}, \mathrm{c}, *}$ \\ a Department of Mechanical Engineering, University of Connecticut, Storrs, CT, USA \\ b New England Sickle Cell Institute, University of Connecticut Health Center, Farmington, CT, USA \\ ${ }^{\mathrm{c}}$ Department of Biomedical Engineering, University of Connecticut, Storrs, CT, USA
}

\section{A R T I C L E I N F O}

Keywords:

Erythrocytes

ICAM-4

$\alpha v \beta 3$

Surface functionalization

Viscoelasticity

Neo-Hookean

\begin{abstract}
A B S T R A C T
Current microfluidic assays, which aim at quantifying mechanical properties of sickle cell red blood cells (SSRBCs), suffer from a number of drawbacks in functionalization and flow control. Specifically, physical adsorption functionalization techniques produce inconsistent functional surfaces, and common volumetric flow pumps cannot be used to adjust the flow inside microchannels with minimal delay. We have designed an experimental setup that alleviates these complications by implementing aspiration for microchannel assembly that enables the use of most functionalization techniques and a pressure controller that allows instant and precise changes in the microchannel flow. Utilizing this setup, we have quantified SS-RBC adhesion to the integrin $\alpha v \beta 3$, a specific adhesion protein expressed on the endothelium, as well as measured the shear modulus and viscosity of the SS-RBC plasma membrane.
\end{abstract}

\section{Introduction}

Sickle cell disease (SCD) is a genetically inherited disorder characterized by painful vaso-occlusive crises in which blood flow to parts of the body is obstructed (Odièvre et al., 2011). Vaso-occlusion is the result of interactions between blood cells and the endothelium (Hebbel et al., 2004; Telen, 2005; Hebbel et al., 2009). It has been shown that the adhesive behavior and viscoelastic response of SS-RBCs are important factors in vaso-occlusion (Evans et al., 1984). SS-RBC adhesion to specific proteins, such as human integrin $\alpha_{v} \beta_{3}$, expressed on the endothelium has been implicated as part of the mechanism that triggers vaso-occlusive crises (Zennadi et al., 2004; Zhang et al., 2017). During a vaso-occlusive crisis adherent SS-RBCs in the vasculature obstruct other SS-RBCs due to their viscoelastic and mechanical properties (Hillery and Panepinto, 2004; Quinn et al., 2011).

A number of experimental tools have been used to quantify SS-RBC adhesion to proteins expressed on the endothelium. To this end, atomic force microscopy (AFM) has been employed in single-molecule force spectroscopy (SMFM) and single-cell force spectroscopy (SCFS) assays (Maciaszek et al., 2012; Maciaszek et al., 2014; Zhang et al., 2017). These assays offer insight into molecular level interactions, but only a few RBCs can be tested this way under each condition. In contrast, perfusion chambers and microfluidic devices have been utilized with the same aims (Hillery et al., 1996; Higgins et al., 2007). These setups provide the potential for concurrent high-throughput assays that are more clinically relevant than AFM-based techniques. However, current microfluidic assays face many design challenges in terms of functionalization and flow control. Specifically, physical adsorption of targeted proteins directly on the microchannel surfaces has become a staple of microfluidic assays due to the ease of application and because of the difficultly in utilizing other strategies when microchannels are assembled using oxidative sealing (McDonald and Whitesides, 2002). Unfortunately, this functionalization technique produces weak and unstable immobilization bonds between the proteins and the substrate. In addition, proteins do not bind specifically, orient themselves randomly, and are capable of forming as many bonds as they have binding sites (Kim and Herr, 2013). This can lead to experimental protocols that are marred by background $\mathrm{RBC}$ adhesion to the substrate itself and adhesion to protein binding sites that are not of interest to the specific study. On the other hand, the use of volumetric flow pumps in microfluidic assays prevents instant changes in flow parameters, thus severely limiting options in assay design.

The mechanical properties of wildtype RBCs have been explored both empirically and in simulations (Hochmuth et al., 1979; Dao et al., 2003; Li et al., 2016). As such, optical tweezers and micro-pipettes have also been employed to study SS-RBC viscoelastic properties (Evans et al., 1984; Mohandas and Evans, 1994). Also, the mechanical properties of SS-RBCs have been investigated using AFM (Maciaszek

\footnotetext{
* Corresponding author at: Department of Mechanical Engineering, University of Connecticut, 191 Auditorium Road, Storrs, CT 06269, USA.

E-mail address: gelyko@engr.uconn.edu (G. Lykotrafitis).
} 
et al., 2011). Because these assays feature direct manipulation of individual SS-RBCs they are time consuming, have low throughput, and contain generalizations and potential errors arising from the type and shape of the device. Tangentially, a microfluidic assay enabled by a custom made pressure driven flow has been used to study viscoelastic properties of sickle cell trait RBCs (Zheng et al., 2015).

In this work, we propose a microfluidic approach that addresses the complications found in adhesion assays by using a functionalization strategy in which a known protein binding site covalently bonds with the substrate in order to create a uniformly dense functionalized surface. In order to facilitate the functionalization, the microchannel is assembled through aspiration, which keeps the functionalized areas inside of the microchannel untouched (Le Berre et al., 2006).

In concert with this design, a pressure controlled pump allows us to introduce a well-controlled flow inside the microchannel. The use of a pressure pump allows us to quantify SS-RBC adhesion to a functionalized substrate not only by the fraction of remaining adherent RBCs at the end of each experiment but also by their ability to withstand wall shear stress increases during experiments. This feature empowers us to quantify the mechanical properties of SS-RBCs by instantly modulating the flow while tracking the morphological changes in SS-RBCs that have adhered directly to a glass substrate.

\section{Materials and methods}

\subsection{Equipment}

Precise and steady control of microfluidic flow was achieved with a pressure controller (OB1 Microfluidic Flow Control System; Elveflow, Paris, France). Both the inlet and outlet ports of the microfluidic device were independently controlled by the pressure controller in order to increase precision. The microchannel was observed and recorded digitally by an Orca-Flash 2.8 high speed camera (Hamamatsu Photonics K.K.; Hamamatsu City, Japan) attached to an Olympus IX73 inverted microscope (Olympus Corporation; Shinjuku, Tokyo, Japan). A diagram of the setup is shown in Fig. 1A. In adhesion experiments a 20x magnification objective lens, numerical aperture (NA) of 0.45 , was used, while recovery experiments utilized a $40 \mathrm{x}$ magnification objective lens, NA of 0.6 . The high speed camera captured 45 frames a second. A custom LabVIEW user interface was

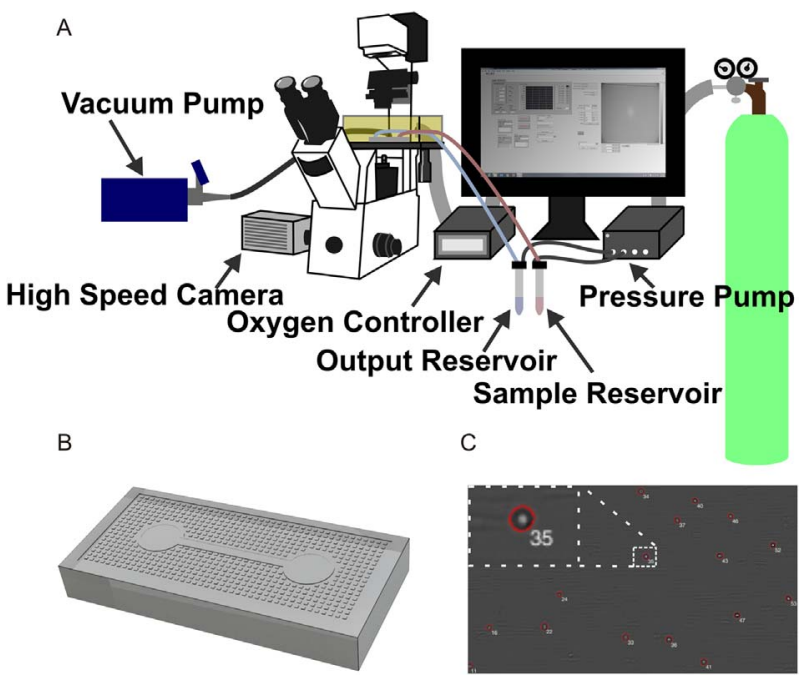

Fig. 1. A. Graphic showcase of the experimental equipment. B. A computer-aided design rendering of the polydimethylsiloxane component of the microfluidic assembly exhibiting the aspiration network around the microchannel. C. A processed background image of a video recorded during an $\mathrm{RBC}$ to endothelial protein adhesion assay where individual adherent RBCs are identified. The paths of non-adherent RBCs can be made out as blurs. used to control both devices digitally and record pressure readings and video footage simultaneously.

\subsection{Microchannel fabrication}

Polydimethylsiloxane (PDMS) microchannels were created using photolithography to give all features a height of approximately $32 \mu \mathrm{m}$. The master mold was created following standardized permanent epoxy negative photoresist processing guidelines provided by MicroChem (USA; Westborough, MA). To summarize briefly, the master mold was created by covering a $100 \mathrm{~mm}$ diameter silicon wafer with SU-8 2025 negative photoresist, spin coated to achieve the desired feature height, covered with the mask of the design, and exposed to ultraviolet (UV) light in order to crosslink the polymer. The relief structure was then developed using 1-Methoxy-2-propyl acetate (SU-8 developer) and covered with PDMS prepolymer, which was then cured to create the PDMS elastomer. The PDMS microchannels were cut from the wafer and peeled off from the master mold. The microchannel device featured a $15 \mathrm{~mm}$ long and $1 \mathrm{~mm}$ wide microchannel, connected at either end to $6 \mathrm{~mm}$ diameter antechamber wells. The size of the wells was necessary to promote structural stability of the microchannel.

The microfluidic device was attached, through aspiration, to a functionalized glass slide substrate (See Fig. 1B) (Le Berre et al., 2006). This method of assembly allows substrates to be functionalized before the device is assembled since the functionalization is not altered or destroyed during assembly, as in oxidative sealing. Truncated and polished 20 gauge needles were employed as the inlet and outlet ports of the microchannel, and were connected to $6 \mathrm{~mm}$ diameter antechamber wells of the microfluidic device. The aspiration network was connected by a $2 \mathrm{~mm}$ diameter tygon tube to a vacuum pump that supplied a negative pressure of $75 \mathrm{kPa}$. The microchannel was brought into contact with the functionalized glass slide substrate while submerged in phosphate buffered saline (PBS), in order to prevent the formation of an air bubble inside the microchannel.

\subsection{Functionalization}

Human integrin $\alpha_{v} \beta_{3}$ (EMD Millipore; Billerica, MA, USA) was covalently bound to an N-hydroxysuccinimide (NHS) functionalized glass slide (NHS surfaces; MicroSurfaces, Inc., Englewood, NJ, USA) in order to facilitate a uniform coating (Kim and Herr, 2013). In order to bind the protein, it was deposited onto the slide at a concentration of $270 \frac{\mathrm{mg}}{\mathrm{mL}}$ in Triton-X 100 and incubated on the slide for $20 \mathrm{~min}$ at $37^{\circ} \mathrm{C}$. The excess solution was then rinsed off the slide with PBS, the slide was coated with a proprietary buffer solution (MicroSurfaces, Inc., Englewood, NJ, USA) to remove unbound NHS groups and incubated for $30 \mathrm{~min}$ at room temperature to this end. The excess buffer solution was rinsed off the slide with PBS. The functionalized slide was then stored in PBS and immediately used.

\subsection{Sample preparation}

This study was approved by the Institutional Review Boards of the UCONN Health Center and UCONN-Storrs. Volunteers with SCD, at least 18 years of age, were eligible to participate in this study. RBCs from consented volunteers were separated from freshly drawn, heparin-anticoagulated, venous blood by centrifuge, at a relative centrifugal force of $500 \mathrm{~g}$ for $10 \mathrm{~min}$ at $4{ }^{\circ} \mathrm{C}$. Afterward, the buffy white coat and plasma were removed by aspiration, and the RBCs were washed three times with Alsever's solution, a saline solution used to store blood (Alsever and Ainslie, 1941). RBCs were diluted down to a hematocrit of $0.5 \%$ for the adhesion assay and $1 \%$ for the viscoelasticity assay in Alsever's solution before being introduced into the microchannel. This hematocrit was selected in order to maximize the acquisition of useful images during video recording, such that there were as many non- 
overlapping RBCs in each frame as possible.

Epinephrine was added to the SS-RBC solution to produce a final concentration of $16.4 n M$. The SS-RBCs solution with Epinephrine was incubated for $30 \mathrm{~min}$ before the experiment was conducted. Propranolol was added to the SS-RBC to produce a final concentration of $10 \mu M$. The SS-RBC solution with propranolol was incubated for $30 \mathrm{~min}$ before the experiment was conducted.

\subsection{Adhesion assay}

SS-RBCs from one concenting patient in Alsever's Solution (SigmaAldrich, St. Louis, MO, USA) at a $0.5 \%$ hematocrit were introduced into the microchannel and allowed to adhere to the functionalized substrate for 10 minutes at a pressure drop that produced a $0.1 \frac{\text { dynes }}{\mathrm{cm}^{2}}$ wall shear stress. After this initial period, all RBCs in a $3 \mathrm{~mm}^{2}$ area inside the microchannel were recorded. The pressure drop across the microchannel was then increased to produce $1,5,10,20$, and $30 \frac{\text { dynes }}{\mathrm{cm}^{2}}$ wall shear stress and the adherent RBCs were recorded in the same $3 \mathrm{~mm}^{2}$ area 1 minute after each increase. After all wall shear stresses measurements were taken, the microchannel was discarded.

\subsection{Viscoelasticity assay}

Untreated, baseline, SS-RBCs from one concenting patient, in Alsever's Solution at a $1 \%$ hematocrit were introduced into the microchannel and allowed to adhere to the glass substrate for $10 \mathrm{~min}$ with no pressure drop across the microchannel. The recoveries of adherent SS-RBCs due to the instant change in wall shear stress from 1 $\frac{\text { dynes }}{\mathrm{cm}^{2}}$ (physiological wall shear stress) to $0 \frac{\text { dynes }}{\mathrm{cm}^{2}}$ were recorded.

\subsection{Data processing}

A custom MATLAB script was used to detect and quantify adherent RBCs. A background image of each recorded video was created by averaging all individual pixel values over all frames. This filtering method obfuscates all moving objects and retains all stationary ones in the background image. The background was then cropped to the area of interest, devignetted, and sharpened using unsharp masking. The edges of all RBCs were quantified by the Canny edge detection algorithm. The convex hulls of all object edges were then labelled, and sorted based on size: objects smaller than a potential RBC (smaller than $4 \mu \mathrm{m}$ in diameter) were discarded while objects larger than a potential RBC (larger than $12 \mu \mathrm{m}$ in diameter) were broken up into multiple objects using the watershed algorithm. The final set of objects were again sorted by size such that objects too small (smaller than $4 \mu \mathrm{m}$ in diameter) or too large to be an RBC (larger than $12 \mu \mathrm{m}$ in diameter) were discarded. The remaining objects were counted and overlaid on the original image, so that the computed result could be double checked and adjusted manually to ensure its accuracy (see Supplmentary Fig. S1).

In viscoelastic experiments, RBCs were identified on a frame by frame basis after the frames were cropped, sharpened, and devignetting. Each individual RBC was identified and tracked throughout a video. The tracking was automated using a custom MATLAB code, and the results were verified manually and corrected where necessary. RBC morphology was determined by fitting the identified shape of the RBC with an ellipse. Only adherent yet freely deformable and mobile RBCs were selected, manually, for processing (see Supplmentary Fig. S2 for an example of SS-RBC morphology at different applied wall shear stresses).

Though these simple MATLAB codes have been highly customized for our use and are of little scientific interest in and of themselves, we have made them available online (http://www.engr.uconn.edu/ \%7Egelyko/additionalsoftware.html) as an example of the exact processing logic, algorithms, and working parameters we used.

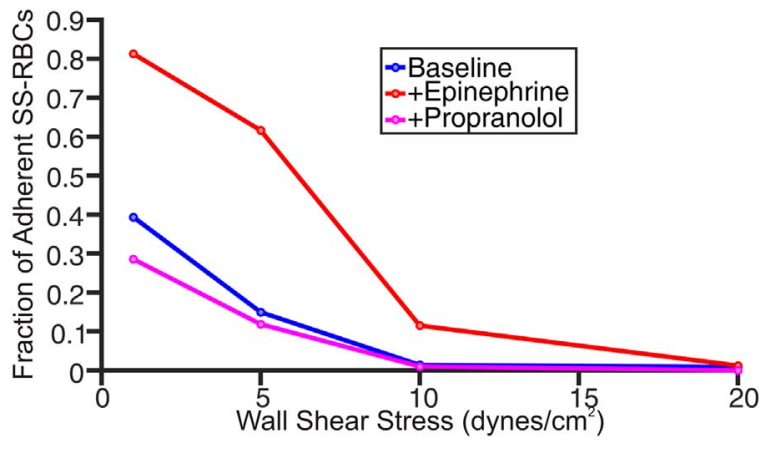

Fig. 2. Adherent populations of untreated SS-RBCs alongside those treated with epinephrine and propranolol relative to their respective initial adherent populations to a substrate coated with human integrin $\alpha_{v} \beta_{3}$ at increasing wall shear stresses. When treated with epinephrine, SS-RBCs adhere in greater relative numbers at larger stress than when untreated. SS-RBCs treated with propranolol appear to adhere in fewer relative numbers than untreated SS-RBCs. Due to the large number of initial SS-RBCs ( $>300$ for each data point), the $95 \%$ confidence intervals of each data point, if displayed, would be obscured entirely by the symbols representing the measured values.

\section{Results and discussion}

\subsection{Adhesion assay}

One patient's SS-RBC's affinity to adhere to a $\alpha_{v} \beta_{3}$ functionalized substrate was modulated by epinephrine, an agonist, which has been shown to increase SS-RBC adhesion to the endothelial protein (Zennadi et al., 2004). Epinephrine produces this effect by upregulating cAMP in RBCs and stimulating protein kinase A, which increases SSRBC adhesion (Telen, 2005). Consistent with previous findings, the adherent population of SS-RBCs treated with epinephrine was not only greater but resisted higher wall shear stresses than SS-RBCs not treated with epinephrine (See Fig. 2). Additionally, we predict that antagonists, such as propranolol, may not only block the action of agonists, but actively reduce SS-RBC adhesion to a $\alpha_{v} \beta_{3}$ functionalized substrate. Consistent with this conjecture, we found that the adherent population of SS-RBCs treated with propranolol was lower and could not withstand wall shear stresses as high as that of untreated SS-RBCs (see Fig. 2).

Each measurement is based on a minimum of 300 initially adherent RBCs spread uniformly over a $3 \mathrm{~mm}^{2}$ area. Background adhesion, evaluated with a slide coated only with the background PEG layer, was found to be negligible below a shear stress of $5 \frac{\text { dynes }}{\mathrm{cm}^{2}}$ and non-existent above (data not shown). Thus, we are quantifying, almost exclusively, SS-RBC adhesion to human integrin $\alpha_{v} \beta_{3}$. By incrementally increasing wall shear stress on the group of adherent SS-RBCs and recording each change, we are able to meaningfully measure the strength of adhesion of SS-RBCs to the specific receptor $\alpha_{v} \beta_{3}$ with high throughput. Due to this uniformity and the large number of processed cells, we expect that the reported values are true for the entire sample and that this precision will remain consistent in future patient samples. As with AFM measurements, we expect to observe large variability between samples drawn from different patients and even samples drawn from the same patient on different dates. However, when we treated the patient sample with epinephrine and propranolol the modulation of the adhesion was in agreement with the expected trend that we have previously reported based on AFM. We expect that using the proposed method we will be able to measure the same modulation in future patient samples.

We note that the adhesion levels of individual RBCs from the same sample are different from cell to cell. This has been observed in whole $\mathrm{RBC}$ adhesion experiments where the adhesion force between a RBC and a functionalized substrate was measured via AFM (Maciaszek et al., 2014) and in single molecule force spectroscopy experiments 

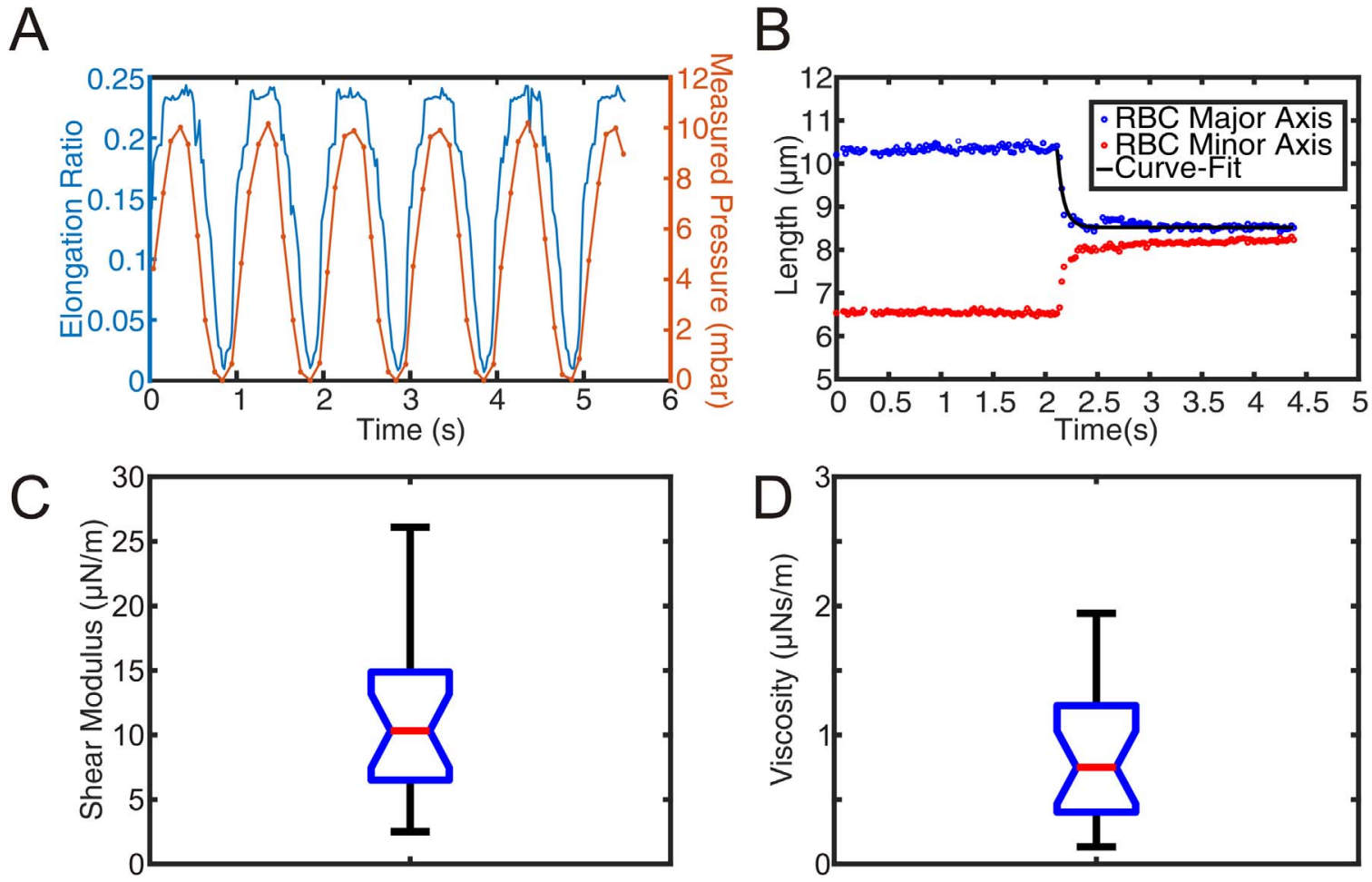

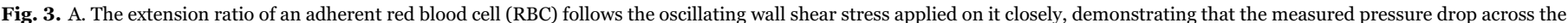

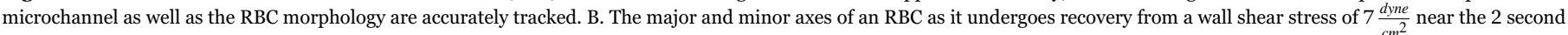

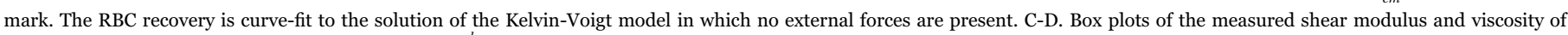
adherent SS-RBCs $(n=21)$ as they recovered from $1 \frac{\text { dyne }}{\mathrm{cm}^{2}}$ wall shear stress. The notches on the box-plots represent the $95 \%$ confidence intervals of the measured medians.

where the expression of active adhesion receptors was measured (Maciaszek et al., 2012; Zhang et al., 2017). Because of this, we expect that the same level of wall shear stress will not have the same effect on all RBCs in terms of detachment from the substrate. Nonetheless, we observed that higher wall shear stress results in a lower adherent fraction and after a sufficiently high value almost, depending on the RBC adhesion level, all RBCs are detached.

\subsection{Continuous flow and RBC elongation}

Based on the pressure pump's internal pressure sensors, which record pressure drop experienced across the microchannel every $0.1 \mathrm{~s}$, we have found that the response time of our microfluidic system is $0.1 \mathrm{~s}$ at the slowest. Observations of RBC morphology suggest that the system responds even faster and clearly indicate that continuous input pressure changes are experienced across the microchannel corresponding to continuous pressure changes. We have verified this by applying an oscillating pressure drop across the channel and simultaneously measuring the major and minor axes lengths of an adherent RBC and evaluating its elongation index as $E I=\frac{\text { major axis }- \text { minor axis }}{\text { major axis }+ \text { minor axis }}$ (see Fig. $3 \mathrm{~A}$ ).

\section{3. $S S-R B C$ recovery}

The viscoelastic properties of the SS-RBC membrane were quantified by measuring the shear modulus and viscosity using the Kelvin-Voigt model for a Neo-Hookean near incompressible material with linear viscous response:

$\frac{T_{s}}{2 \mu}=\frac{1}{4}\left(\lambda^{2}-\lambda^{-2}\right)+t_{c} \frac{\partial \ln (\lambda)}{\partial t}$

where $\mu$ is the membrane shear modulus, $t_{c} \equiv \frac{\eta}{\mu}$ is the characteristic time, $\eta$ is the membrane viscosity, $\lambda=\frac{\text { deformedmajoraxisRBClength }}{\text { undeformedmajoraxisRBClength }}$ is the extension ratio, and $T_{s}=\frac{T_{1}-T_{2}}{2}$ is the membrane shear stress where
$T_{1}=\frac{\tau * \text { deformRBCarea }}{\text { deformedRBCminorlength }}$ and $T_{2}=0$ are the in-plane principal membrane stresses, and $\tau$ is the wall shear stress applied on the RBC (Hochmuth et al., 1979; Mills et al., 2004). The shear modulus was obtained via the expression $\mu=\frac{2 T}{\left(\lambda^{2}-\lambda^{-2}\right)}$, where $\lambda$ is the extension ratio between two steady-state conditions. The viscosity was evaluated as $\eta=\mu^{*} t_{c}$ where $t_{c}$ was found by curve fitting the relaxation data of the instantaneous extension ratio $\lambda(t)$ with the analytical solution $\lambda(t)=\sqrt{\frac{\Lambda+\exp \left(-t / t_{c}\right)}{\Lambda-\exp \left(-t / t_{c}\right)}}$ of the governing equation for zero applied membrane forces (see Fig. 3B), where $t$ is the time, $\Lambda=\frac{\lambda_{m}^{2}+1}{\lambda_{m}^{2}-1}$, and $\lambda_{m}$ is the extension ratio at the instance when the applied membrane forces are set to zero.

The viscosities and shear moduli of SS-RBCs were measured using this method as shown in Figs. 3C and D. The measured shear moduli are of the same order of magnitude, though nominally higher, as those reported by (Mills et al., 2004). This is consistent with our expectations, since the aforementioned work investigated wild type RBCs and previous efforts from our lab have measured higher Young's Moduli in SS-RBCs with respect to their wild type RBC counterparts (Maciaszek and Lykotrafitis, 2011). The time constant, $t_{c}$, was found to be on the same order of magnitude as the original findings by (Hochmuth et al., 1979), signifying that the reported viscosity measurements are in agreement with both works. The width of the $95 \%$ confidence of the shear modulus measurements $\left(\sim 2 \frac{\mu N}{m}\right)$ is a product of the large number of processed RBCs, and we expect future processed samples to continue to produce measurements of comparable precision.

\subsection{Conclusion}

The implemented experimental setup presents an opportunity to quickly and precisely quantify the mechanical and pharmacological properties of SS-RBCs in many different assays. This is enabled by the combination of equipment, specifically the high-speed camera and the 
precise and instantly modifiable pressure flow, the surface functionalization protocol, and custom image processing software. Minor tweaks to the assay parameters, such as the concentration of endothelial protein during functionalization, may also be appropriate to maximize the sensitivity of the system and elucidate SS-RBC properties. The current setup can be easily modified to enable experiments under controlled oxygen levels.

These experimental methods may potentially also be conducted in similar assays on wildtype RBCs or in efforts to study other related diseases, such as malaria or diabetes, where either RBC adhesion or mechanical behavior plays a major role in pathology. The same advantages and improvements presented in this report may prove to be applicable is such studies. Thus, this potential is an avenue for future work in this area.

\section{Funding}

This work was supported by the Connecticut Institute for Clinical and Translational Science (CICATS) at the University of Connecticut and UCONN Academic Plan II. The content is the sole responsibility of the authors and does not necessarily represent the official views of CICATS. GL is supported by the National Science Foundation (CMMI-1235025, PHY-1205910), the American Heart Association (12SDG12050688).

\section{Competing Interests}

None.

\section{Acknowledgements}

We thank Ms. Kaylee Perron for her assistance in processing and acquiring preliminary recovery data and her input in improving portions of the video processing code.

\section{Appendix A. Supplementary material}

Supplementary data associated with this article can be found in the online version at doi:10.1016/j.jmbbm.2017.02.031.

\section{References}

Alsever, J.B., Ainslie, R.B., 1941. A new method for the preparation of dilute blood plasma and the operation of a complete transfusion service. NY State J. Med. 41, 126-131.
Dao, M., et al., 2003. Mechanics of the human red blood cell deformed by optical tweezers. J. Mech. Phys. Solids 51 (11), 2259-2280.

Evans, E., et al., 1984. Static and dynamic rigidities of normal and sickle erythrocytes. Major influence of cell hemoglobin concentration. J. Clin. Investig. 73 (2), 477.

Hebbel, R.P., et al., 2004. The endothelial biology of sickle cell disease: inflammation and a chronic vasculopathy. Microcirculation 11 (2), 129-151.

Hebbel, R.P., et al., 2009. A systems biology consideration of the vasculopathy of sickle cell anemia: the need for multi-modality chemo-prophylaxis. Cardiovasc. Haematol. Disord.-Drug Targets (Former. Curr. Drug TargetsCardiovasc. Hematol. Disord.) 9 (4), 271-292.

Higgins, J., et al., 2007. Sickle cell vasoocclusion and rescue in a microfluidic device. Proc. Natl. Acad. Sci. 104 (51), 20496-20500.

Hillery, C.A., et al., 1996. Increased adhesion of erythrocytes to components of the extracellular matrix: isolation and characterization of a red blood cell lipid that binds thrombospondin and laminin. Blood 87 (11), 4879-4886.

Hillery, C.A., Panepinto, J.A., 2004. Pathophysiology of stroke in sickle cell disease. Microcirculation 11 (2), 195-208.

Hochmuth, R., et al., 1979. Red cell extensional recovery and the determination of membrane viscosity. Biophys. J. 26 (1), 101.

Kim, D., Herr, A.E., 2013. Protein immobilization techniques for microfluidic assays. Biomicrofluidics 7 (4), 041501.

Le Berre, M., et al., 2006. Reversible assembling of microfluidic devices by aspiration. Microelectron. Eng. 83 (4), 1284-1287.

Li, X., et al., 2016. Patient-specific blood rheology in sickle-cell anaemia. Interface Focus 6 (1), 20150065.

Maciaszek, J.L., et al., 2012. Epinephrine modulates BCAM/Lu and ICAM-4 expression on the sickle cell trait red blood cell membrane. Biophys. J. 102 (5), 1137-1143.

Maciaszek, J.L., et al., 2011. Microelasticity of red blood cells in sickle cell disease. J. Strain Anal. Eng. Des. 46 (5), 368-379.

Maciaszek, J.L., Lykotrafitis, G., 2011. Sickle cell trait human erythrocytes are significantly stiffer than normal.. J. Biomech. 44 (4), 657-661.

Maciaszek, J.L., et al., 2014. Single-cell force spectroscopy as a technique to quantify human red blood cell adhesion to subendothelial laminin. J. Biomech. 47 (16), 3855-3861.

McDonald, J.C., Whitesides, G.M., 2002. Poly (dimethylsiloxane) as a material for fabricating microfluidic devices. Accounts Chem. Res. 35 (7), 491-499.

Mills, J., et al., 2004. Nonlinear elastic and viscoelastic deformation of the human red blood cell with optical tweezers. MCB 1, 169-180.

Mohandas, N., Evans, E., 1994. Mechanical properties of the red cell membrane in relation to molecular structure and genetic defects. Annu. Rev. Biophys. Biomol. Struct. 23 (1), 787-818.

Odièvre, M.-H., et al., 2011. Pathophysiological insights in sickle cell disease. Indian J. Med. Res. 134 (4), 532.

Quinn, D.J., et al., 2011. Combined simulation and experimental study of large deformation of red blood cells in microfluidic systems. Ann. Biomed. Eng. 39 (3), 1041-1050.

Telen, M.J., 2005. Erythrocyte adhesion receptors: blood group antigens and related molecules. Transfus. Med. Rev. 19 (1), 32-44.

Zennadi, R., et al., 2004. Epinephrine acts through erythroid signaling pathways to activate sickle cell adhesion to endothelium via $L W$-av $\beta 3$ interactions. Blood 104 (12), 3774-3781.

Zhang, J., et al., 2017. Regulation of active ICAM-4 on normal and sickle cell disease RBCs via AKAPs is revealed by AFM. Biophys. J. 112 (1), 143-152.

Zheng, Y., et al., 2015. Mechanical differences of sickle cell trait (SCT) and normal red blood cells. Lab Chip 15 (15), 3138-3146. 